Oliwko Elżbieta, Babuśka-Roczniak Magdalena, Wojtanowska-Kaczka Magdalena, Lyubinets Oleh, Roczniak Wojciech. Physiotherapeutic recommendations for patients with cystic fibrosis. Journal of Education, Health and Sport. 2020;10(11):96-103. eISSN 2391-8306. DOI http://dx.doi.org/10.12775/JEHS.2020.10.11.010

https://apcz.umk.pl/czasopisma/index.php/JEHS/article/view/JEHS.2020.10.11.010

https://zenodo.org/record/4278924

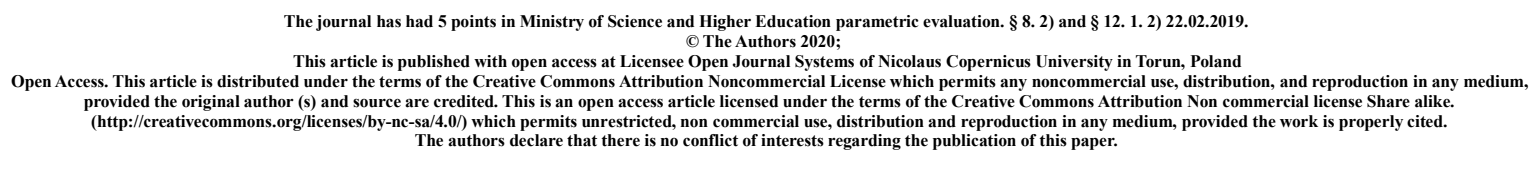

Received: 03.11.2020. Revised: 11.11.2020. Accepted: 18.11.2020.

\title{
Physiotherapeutic recommendations for patients with cystic fibrosis
}

\author{
Elżbieta Oliwko', Magdalena Babuśka-Roczniak' ${ }^{1}$, Magdalena Wojtanowska-Kaczka ${ }^{1}$, \\ Oleh Lyubinets ${ }^{2}$, Wojciech Roczniak ${ }^{1}$
}

${ }^{1}$ The Jan Grodek State University in Sanok, Medical Institute, Poland
${ }^{2}$ Danylo Halytsky Lviv National Medical University, Lviv, Ukraine

Abstract Cystic fibrosis is a genetically determined disease. The development of medicine has resulted in an improvement and prolongation of life. Numerous scientific studies support the thesis that maintaining physical performance is a key element of complex treatment. The basis of treatment should be: parallel pharmacological treatment and physiotherapeutic. The aim of this work was to present the physiotherapeutic techniques used in this disease. The main methods of physiotherapeutic treatment include positional drainage, forced exhalation technique, active breathing cycle technique, autogenous drainage, variable bronchial pressure technique and increased respiratory pressure technique. Physiotherapy of the respiratory system is of major importance, the main aim of which is to prevent the deterioration of the already existing respiratory failure. Each of the presented methods of physiotherapy was created thanks to constant search for the perfect technique to cleanse the bronchial tree. This disease has a different clinical course and each technique should be adapted to the individual needs of the patien and the basis of effective therapy is systematic, daily cleansing of the bronchial mucus from the bronchial tree. Physiotherapy applied earlyIn the thoracic region, it is the treatment of choice for patients with cystic fibrosis improves the quality of life and prolongs the patient's age.

Conclusions:

1. There is no effective, causal treatment for cystic fibrosis, and drug treatment comes down to treating complications and often has side effects.

2. Comprehensive physiotherapy is the golden mean in the treatment of cystic fibrosis and significantly prolongs the life of patients.

3. The effectiveness of physiotherapy depends on the time of commencement, regularity and personalization of treatments.

Key words: cystic fibrosis, treatment methods, physiotherapy. 


\section{Introduction}

Cystic fibrosis (CF) is a chronic disease genetically determined [2,23]. It was described as a separate disease entity over 50 years ago, but the first mentions of this disease appeared in the 17 th century. The prevalence of this disease in the population is $1 / 3000$ people, while the carriers are much more, i.e. 1 in 20 people, therefore it belongs to the most frequently inherited genetic defect mainly in North America, Australia and Europe.

Cystic fibrosis belongs to the genetic diseases known as channelopathies, in which they come and to mutating a gene encoding a membrane transport protein of a particular ion. In cystic fibrosis, this is a mutation in the gene responsible for the synthesis of the membrane chloride channel (CFTR - cystic fibrosis transmembrane conductance regulator). This mutation is inherited autosomal and recessively, the child will become ill when he receives this gene from both parents. The effect of abnormal function or lack of this protein is disruption of chloride ion transport through cell membranes [8]. This causes a thickening and wear of the mucous viscosity in the lungs and other organs. The consequence of the accumulation of secretions in the ducts is the malfunction of the mucous glands in the lungs, gastrointestinal tract and other organs. In the lungs, normal tissue is replaced by cystic fibrosis.

The development of modern medicine resulted in a significant extension of life, in $193870 \%$ of patients died in the first year of life. In the early 1990s, the average age in highly developed countries fluctuated as around 30 years. Currently, it is stated that the life expectancy of children born with this disease will be at least 40 years. Once the disease was the domain of pediatricians, now this disease is dealt with by a team of specialists due to its appearance dysfunction many organs over the years of the disease $[2,8,14,23]$. The intensity of changes determines the length and quality of life of patients. The disease appears in infancy the main symptoms of the disease are: chronic cough, recurrent respiratory infections, recurrent pancreatitis, meconium obstruction, fatty stools, emaciation or lack of weight gain despite a very good appetite. These symptoms vary in its intensity in individual patients, it mainly concerns the degree of involvement lungs and pancreas [6,12,22]. It's known that is it's addicted on the degree of mutation the gene encoding CFTR, responsible for the occurrence of the disease as well as its occurrence additional non-genetic factors [4,24].

In the classic full-blown form, there are:

- bronchitis and pneumonia

- pancreatic exocrine insufficiency

- increased chloride concentration in sweat 
sterility

The quality and life expectancy is determined primarily by changes in the respiratory system, usually we are dealing with a chronic infection and periods of exacerbation accompanied by acceleration of breathing, cough and fever [21,22]. Recurrent infections lead to gradual lung damage, hence to respiratory failure, which is often the direct cause of the death of these sick people.

In the gastrointestinal tract, sticky mucus clogs the pancreatic ducts and this causes gradual damage to this organ. These changes may appear in utero, but in most cases the functional impairment is more pronounced for years. As the pancreas secretes enzymes and bicarbonates necessary for proper digestion of food, its abnormal function in this disease causes digestive disorders in children [22,23]. Due to the enzymatic disturbance of food that is insufficiently decomposed and absorbed, children are malnourished and their growth and physical development are largely limited. Undigested portions of the food result in smelly, fatty stools and chronic diarrhea. In advanced stages of the disease damage occurs pancreatic islets and insulin deficiency and the development of diabetes mellitus. In some patients, changes in the common bile duct can be found, which results in cholestasis and cirrhosis $[16,22]$. Meconium obstruction is another disturbance of the digestive system, encountered in the first 24 hours of a child's life, consisting in obstruction of the small intestine with sticky meconium. The symptoms of this condition are gas, vomiting and your child's general condition which deteriorates very quickly, requiring immediate attention surgical intervention.

Cystic fibrosis is often the cause of infertility. Women produce abnormal mucus in the cervix and have irregular menstrual cycles. Even when fertilization occurs, pregnancy carries a very high risk. It is known that good lung function in the mother gives a good chance of giving birth to a healthy child, while the problem is the increased probability of the disease in the offspring, even with the correct course.in pregnancy and childbirth. In men, on the other hand, we find the complete absence or underdevelopment of the vas deferens, the consequence of which is infertility.

The changes in organs are accompanied by delayed physical development, and at the same time deficiency in the patient's body weight and height. Developmental impairment and atrophy of large muscle groups are noted. Another symptom directly related to a poorly developed muscular corset is quick fatigue during exercise.

\section{Classification of cystic fibrosis}

The international ICD classification is based on changes in individual organs: 
1. CF with respiratory symptoms (E84.0)

2. CF with gastrointestinal symptoms (E84.1)

3. CF with other organ symptoms (E84.8)

4. CF undefined (E 84.9)

Single isolated symptoms that occur in the patients are not classified as cystic fibrosis and belong to the group of diseases of independent CFTR mutations and they are: sclerosing cholangitis, transient neonatal hypertrypsinogenemia, chronic pancreatitis, disseminated bronchiectasiswhether obstructive azoospermia.

The diagnosis of cystic fibrosis is based on:

a) changes in the respiratory system through: physical examination, performance radiological examination (X-ray, computed tomography)

b) the result of the sweat test documenting the increased excretion of chlorine and sodium ions with a concentration in the sweat ranging from $60 \mathrm{mmol} / 1$ to 140 $\mathrm{mmol} / \mathrm{l}$ confirms cystic fibrosis is a result of $20-40 \mathrm{mmol} / \mathrm{L}$ for infants and 20 60 for older children). Chloride level testing should be carried out at least twice in specialized laboratories that perform these tests at least 100 times a year for the result to be reliable

c) performing a genetic test for the presence of mutations within CFTR genes

d) family history of the disease among relatives

e) the diagnosis of exocrine pancreatic insufficiency manifested by a syndrome of malabsorption mainly of fats and proteins

Ultimately, the condition for the diagnosis of cystic fibrosis is to find at least three of these components, with the decisive factor being mutations in the CFTR gene on two homologous chromosomes.

\section{Treatment methods}

Pharmacological and physiotherapeutic treatment in the course of the disease requires, first of all, the removal of thick and sticky secretions from the respiratory tract and control of bronchitis and pneumonia with targeted antibiotic therapy [18,23]. Removal of purulent discharge requires administration of mucolytic drugs and physiotherapy of the respiratory system. The use of comprehensive treatment is to slow down irreversible changes in the respiratory system. 


\section{Pharmacological treatment:}

Antibiotics are used in the case of new infections and exacerbation of chronic inflammatory changes in the lungs. In chronically ill patients, the appearance of an exacerbation is strictly defined and refers to an increase in cough and an increase in the amount of expectorated secretion, dyspnoea, wheezing, and new auscultatory and radiological changes. If an exacerbation is detected, it is an indication to start targeted 14-day intravenous antibiotic therapy. In such cases, permanent peripheral access to the vein, the so-called venous port. Only in less severe cases is oral antibiotic therapy allowed. The choice of drugs should be dependent on cultured pathogens in bronchial secretions and performed antibiogram.

It is known from the experience of clinicians that good effects were obtained with the use of macrolides, e.g. azithromycin, due to the duration of action and the bacteriostatic effect of this an antibiotic [18, 20].

Mucolytic treatment it is used to thin and remove viscous secretions, some patients benefit from inhalation with $\mathrm{N}$-acetylcysteine or ambroxol. $\mathrm{NaCl}$ solutions of $3-10 \%$ in the volume of 4-5 ml per inhalation effectively support the evacuation of secretions. Administration of inhaled bronchodilators prior to inhalation is recommended.

Bronchodilator treatment it is most often short and long-acting beta-mimetics that are used in patients with reversible bronchial obstruction. Short-acting drugs are usually used before planned physical exertion. At the time of their application it is necessary effectiveness control using spirometry [10].

Treatment with glucocorticoids it is used in the treatment of bronchial hyperreactivity or asthma. Prednisone is most commonly used as the mainstay of treatment.

Anti-inflammatory treatment indications for this type of chronic treatment should be established by a specialist center that controls the proper treatment of patients with cystic fibrosis. It is used mainly in the non-steroidal anti-inflammatory drug ibuprofen, which when administered in high doses gives a good therapeutic effect.

Gastrointestinal symptoms can be alleviated by administering pancreatic enzymes in the form of tablets. The supplementation of fat-soluble vitamins is very important: A, D, E, K [16]. 
Aerosol therapy used in patients with pulmonary cystic fibrosis is a suitable form of drug administration directly into the bronchus, mucolytics, broncholytics, steroids and even some antibiotics can be administered by this route. Properly conducted therapy should include the appropriate selection of the drug and nebulizer.

Equipment adapted to many months of use should have regularly replaced filters, compressors used on the market in the treatment of cystic fibrosis are those that generate an air flow of 4-8 $1 /$ min. It is recommended to use mouthpieces, which increase the drug delivery to the lungs. The mask is allowed to be used by young children, but the mouthpiece should be changed as soon as possible. The equipment should be replaced after use for a period of 12 months. They must be washed and disinfected after each use. The first administration of a new drug should take place in the presence of a physician due to the possibility of adverse events, including bronchospasm [10]. It is recommended to perform a spirometry test, and if you feel unwell, the treatment should be stopped.

\section{Causal treatment}

The pharmacological treatment methods discussed above are treatment symptomatic. Because cystic fibrosis belongs to genetic diseases in some centers around the world gene therapy $[1,9]$ and causal treatment of CF have been attempted. For now, this therapy is at the research stage and has not been used in the clinic. But That it raises a lot of controversy due to the fact that the carrier of the CFTR gene administered with the nebulizer for the bronchi virus and research is being done in children.

In 2012, it was allowed in the United States for medical use ivacaftor, aplater lumacaftor, which is given in combination with the oral medicine orkambi. This drug affects the CFTR protein and restores the chloride channel. In some countries, the combination of ivacaftor and teracaftor in Symdeko is currently used in CF therapy. .Preparation These give an improvement in those forms of the disease where the chloride channel structure is preserved [3, 19]. Production of these drugs are extremely expensive therefore, due to their price, they will not be universally applicable.

Lung transplant is performed in patients at the end of the disease, when all other methods of treatment are ineffective [7]. In Poland, the first transplant was performed in 2003 and the number of successful transplants has been growing ever since. 


\section{Objective of the work}

The above methods pharmacological treatment is not very effective in the treatment of cystic fibrosis, therefore physiotherapy is an extremely important and parallel therapeutic procedure in this disease. In this work it was decided to present and describe the most important techniques of physiotherapeutic used in this disease.

\section{Physiotherapeutic treatment:}

Physiotherapy should be used regularly in the patient from the moment of diagnosis, even in those who do not have respiratory symptoms. It should be used throughout the patient's life and treated as a compulsory hygienic procedure that improves the health of the individual. Taking into account that the disease lasts for many years, it should be planned and modified with particular care taking into account the patient's age, stage and the period of the disease in which is located $[6,14,15]$. The main principles that apply when using physiotherapy are, first of all, the systematics of performing physiotherapy treatments and exercises, introducing active forms of respiratory physiotherapy, as far as the patient's age allows, and developing self-discipline through learning, e.g. self-draining. It is important that the therapy session is conducted as soon as the child wakes up, before breakfast and after returning from school. It should be varied and correlated with the needs of individual patients, and the time that should be spent on physical therapy should be dependent on respiratory efficiency. Due to the fact that these treatments are part of everyday life and become monotonous, they should be made more attractive by introducing elements of games and movement games. Modern physiotherapy is focused on the use of techniquesallowing the relaxation, displacement and evacuation of secretions from the bronchial tree.

Standards of care should concern the monitoring of the psychomotor development of young children and of physical development in preschool and school age. A great deal of emphasis is placed on educating the parents and family of a child suffering from cystic fibrosis [5,23]. It must be continued throughout the patient's life to improve the quality and length of life and to shape proper pro-health attitudes. 


\section{Physiotherapeutic methods and techniques used in people suffering from cystic fibrosis}

are:

\section{Postural drainage}

One of the first methods used was postural drainage, widely used in Poland since the 1950s. In the majority of patients, it is used as the basic form, with time it has been improved by introducing techniques supporting the drainage effect, such as tapping, shaking, vibration and compression of the lower parts of the chest. For many years it was a standard in the treatment of patients. As the name suggests, the postural drainage consists in arranging the patient in such a way that the bronchus that drains a given segment of the lung is perpendicular to the ground $[5,13,14,17]$. As a result of this arrangement, the secretion by the force of gravity can move from the small bronchioles to the central ones with a large cross-section, from where it is expelled by coughing. In physiotherapeutic practice, 6 main positions are used:

$\checkmark$ the patient lies on his stomach with his head down, the torso inclination to the ground is about 45 degrees, in this position the lower lobes of both lungs are drained, the inclination can be obtained by placing blocks under the rear legs of the bed, or by placing the patient on a special bed for drainage or a trestle

$\checkmark$ the patient lies on the left side with his head down, the torso inclination in relation to the ground is about 30 degrees, in this position the right middle lobe is drained

$\checkmark$ the patient is lying on the right side with the torso rotating backwards and the head facing down, the torso inclination in relation to the ground is about 30 degrees, in this position the left lung tongue is drained

$\checkmark$ the patient lies on his back, head down, torso inclination to the ground about 30 degrees, in this position the basal anterior segments of both lower lobes are drained

$\checkmark$ the patient is lying on his back, the anterior segments of both upper lobes are drained

$\checkmark$ the patient assumes a sitting position with a slight tilt of the torso forward in this position drains the posterior segments of both upper lobes

The above items should be used daily in order to properly perform the drainage procedure, only in infants it is allowed to perform the procedure in a reduced number position. The purpose of techniques that aid drainage is to improve the movement of secretions, reduce respiratory work by passive expiratory support, improve chest mobility, and change the respiratory path through manual inspiratory aids.Patting is done with a folded hand in the so- 
called small boat, they are performed directly over the segment which, in a given body position, has a high chance of cleansing [5,6,17]. The intensity must be adjusted individually to the patient's needs. The use of oppression should be performed with particular care during prolonged exhalation, they are used in the lower parts where the chest is more flexible. Vibrations can be performed with the therapist's hand, however, it is recommended to perform them with the help of a therapist's mechanical devices. The patient's preferences should be taken into account when selecting particular methods. It should be remembered that after draining, the secretion that has flowed from the respiratory tract should be coughed up. The way to do this is to induce spontaneous laughter echoing or inducing a cough reflex by irritating the base of the tongue or by pressing on the laryngeal cartilages. The drainage time for infants should be around 5-10 minutes, while for older children it should be 20-30 minutes. It should be performed once a day and during periods of exacerbation of the disease up to 2 to 3 times a day $[5,12,13]$. It is systematically carried out and is recommended for patients who do not use additional forms of physiotherapy. However, selecting even the best physiotherapeutic methods will not achieve the desired effect if it is not performed regularly.

\section{The technique of forced exhalation}

It consists of making one or two exhalations in combination with calm, controlled breathing. The forceful exhalation phase is performed by the open mouth saying "h". Long exhalations with a low lung volume allow the residual secretions to be removed from the small bronchi and the secretion displaced into the lobar bronchi is removed successively by intensive exhalation $[5,11,15,17]$. It may be an independent drainage method, but it is primarily part of the active respiratory cycle.

\section{The technique of the active breathing cycle}

It consists in performing subsequent activities that create a closed breathing cycle consisting of controlled breathing, thoracic exercises and forced exhalation. Controlled breathing is otherwise called diaphragmatic breathing while the muscles of the shoulder girdle and upper chest are relaxed. Easy breathing rests the bronchial muscles after heavy exhalation and allows you to relax. Thoracic exercise is a way of lower costal breathing. It consists in deep breathing -calm inhalation and a long prolonged exhalation. This method is very important because when you breathe in gently, as the volume of the lungs increases, the resistance to air flow decreases and the connections in the less ventilated parts of the lungs open. This causes air to enter beyond the residual mucus, during exhalation, the mucus is 
broken up and shifted towards the large bronchi $[6,11,12,13,15]$. Air holding may be used in some patients for about 3 seconds at the end of exhalation this will increase the airflow effect. These exercises are performed in conjunction with shaking and patting the chest. The technique should not be used in infants and patients with advanced emphysema. The active respiratory cycle technique can be performed using PNF (proprioceptive neuromuscular facilitation) priming.

\section{Autogenous drainage}

It is based on specific breathing, the purpose of which is to relax and shift the secretions from the small bronchi to the large ones. This technique requires full breath control. This technique uses three phases of breathing:

$\checkmark$ phase I low, the patient continues to exhale until a reserve expiratory volume is achieved

$\checkmark$ phase II mean the patient breathes within the limits of the deepened tidal volume

$\checkmark$ high phase III exhales rapidly and is then expectorated

To obtain the maximum effect, the air must reach the smallest bronchioles, therefore it is recommended to inhale slowly in small portions and stop after each portion. air for 3 seconds $[15,17]$. Autogenic drainage requires good cooperation between the patient and the physiotherapist, therefore it is suitable for older children and adolescents, while for younger children it requires many simplifications. Drainage is performed in the supine position with bent knees or sitting with straight back. The treatment should take approximately 30 minutes.

\section{The technique of variable bronchial pressure}

It is performed with the Flutter apparatus, which consists of a mouthpiece shaft and a metal ball which, under the influence of exhaled air, begins to vibrate, closing and opening the device inlet. Pressure changes in the airways, which causes the bronchus to vibrate and breaks up the secretions, facilitating its evacuation. The amount of resistance can be adjusted by setting the apparatus vertically - it increases the resistance, while the more horizontal one reduces it. The patient should be sitting with elbows on the table, breathe in with a 3 -second pause at the top of the table, then breathe out long and calmly, usually 8 exhalations are recommended [15]. Increasing the number of repetitions can cause hyperventilation. Exercising with this device should not cause too much effort, if that happens, reduce the number of repetitions. 


\section{The technique of increased expiratory pressure PEP (positive expiratory pressure).}

It consists in breathing through an anesthetic type mask with a valve that creates resistance during exhalation. Performing an exhalation with resistance raises the pressure in the bronchi, the pressure gauge determines the exhalation pressure which should range from 10 to 20 meters of water. During the procedure, the patient takes a sitting position with elbows on the table, performs about 8 to 10 breathing cycles, followed by two intense exhalations. However, using this technique may cause right ventricular overload, manifesting as hypoxia. Symptoms of this condition include facial redness, filling of the jugular veins and an increased respiratory frequency $[11,14,15]$. In addition, the mask in patients with bronchial hyperreactivity may induce spasm, therefore bronchodilators should be administered prior to exercise using this technique.

\section{Moderate physical activity}

The essential component of physiotherapy is physical training in the form of general exercise condition. It should be remembered that the tolerance of exercise intensity is variable, it depends on the period of the disease in which the patient is currently located. In the periodsFor exacerbations, effort should be small and carefully dosed according to the performance sick. During periods of remission, exercise duration and intensity can be increased. Very often, patients are diagnosed with muscle weakness, disturbances in body statics and nutritional impairment. When it comes to general development exercises, it is recommended that you take it reduce strength and muscle mass, and exercises that stretch the muscles of the back, chest and shoulder girdles. The best form of physical activity for these patients is cycling and swimming, which facilitates the expectoration of secretions. ZwaLosing children from physical education lessons is justified only in cases of exacerbation of the disease $[15,17,23]$. Regularly used exercises give a significant chance to improve the quality of life and delay the disease progression.

The essence of the effective treatment is adequately early diagnosis of cystic fibrosis, therefore it is extremely important to have a well-collected family history and perform diagnostic tests, including genetic tests, even in the prenatal period.Then, physiotherapeutic treatment can be started from the first days of life.

\section{Summary}

Cystic fibrosis is a genetically determined disease. The development of medicine has resulted 
in an improvement and prolongation of life. Numerous scientific studies support the thesis that maintaining physical performance is a key element of complex treatment. The basis of treatment should be: parallel pharmacological treatment and physiotherapeutic. The aim of this work was to present the physiotherapeutic techniques used in this disease. The main methods of physiotherapeutic treatment include positional drainage, forced exhalation technique, active breathing cycle technique, autogenous drainage, variable bronchial pressure technique and increased respiratory pressure technique. Physiotherapy of the respiratory system is of major importance, the main aim of which is to prevent the deterioration of the already existing respiratory failure. Each of the presented methods of physiotherapy was created thanks to constant search for the perfect technique to cleanse the bronchial tree. This disease has a different clinical course and each technique should be adapted to the individual needs of the patient and the basis of effective therapy is systematic, daily cleansing of the bronchial mucus from the bronchial tree. Physiotherapy applied earlyIn the thoracic region, it is the treatment of choice for patients with cystic fibrosis, improves the quality of life and prolongs the patient's age.

\section{Conclusions:}

1. There is no effective, causal treatment for cystic fibrosis, and drug treatment comes down to treating complications and often has side effects.

2. Comprehensive physiotherapy is the golden mean in the treatment of cystic fibrosis and significantly prolongs the life of patients.

3. The effectiveness of physiotherapy depends on the time of commencement, regularity and personalization of treatments.

\section{Literature:}

1. Alton E., Amstrong D., Ashby Di et al .: Repeated nebulization of non-viral CFTR gene therapy in patients with cystic fibrosis: a randomized, double blind, placebocontrolled, phase 2b trial. Lancet Resp Med. 2015, 3 (9), 684-691.

2. Bal - Bocheńska M., Wolan A., Kwolek A .: Assessment of the effectiveness of airway clearance in children suffering from cystic fibrosis. Medical Review URz, 2009, 1: 3136.

3. Burgener EB, Moss R.: Cystic fibrosis transmembrane conductance regulator modulators: precision medicine in cystic fibrosis. Curr Opin Pediatr., 2018, 30 (3), 372-377. 
4. Castellani C., Assael BM: Cystic fibrosis and clinical review. Cell Mol Live Sci, 2017, 74 (1), 129-140.

5. Demczyszak I., Kuciel-Lewandowska J., Paprocka-Borowicz M .: Physiotherapy in respiratory diseases. In Górnicki. 2009, 31-33.

6. Dolecki W., Rongies W .: Rehabilitation in obstructive airway diseases. Therapy, 2003, 2.

7. Domeńska $H$.: Lung transplants in patients with cystic fibrosis. Borgis, Advances in medical science, 2008, 9,601-605.

8. Elborn JS: Cystic fibrosis. Lancet, 2016, 19, 388 (10059), 2519-2531.

9. Gill DR, Hyde SC: Delivery of genes into the CF airway. Thorax, 2014, 69, 962-964.

10. Gutowski P .: Functional tests of the respiratory system in children. Borgis, Advances in medical science, 2008.9, 559-564.

11. Hoo ZH, Daniels T., Wildman NJ ad all: Airway clearance techniques used by people with CF in the UK. Physiotherapy 2015, 101 (4), 340-348.

12. Małolepsza K : Physiotherapy in children suffering from cystic fibrosis. New Pediatrics, 2000, 5, 14-15.

13. McIIwaine MP, Lee Son NM, Lynn Richmond M .: Physiotherapy and cystic fibrosis: what is the evidence base. Curr Opin Pulm Med. 2014, 20, 613-617.

14. Orlik T .: Evaluation of the effectiveness of selected methods of chest physiotherapy used in the treatment of patients with cystic fibrosis. Med. Developmental Age. 2000: 3: $233-245$.

15. Orlik T., Malinowski A., Skorupa W. et al .: Long-term model of physiotherapeutic treatment in cystic fibrosis. Position of the Polish Working Group. Matio, 2003, 4, 4-9.

16. Rachword TL, Teckman JH, Patel DR: Gastrointestinal pathophysiology and nutrition in cystic fibrosis. Expert Rev Gastroenterol Hepatol. 2018, 12 (9), 853-862.

17. Rosławski A., Woźniewski M .: Respiratory physiotherapy. AWF Wrocław. 2001, 4457.

18. Saimon L., Schechter M .: Evaluating Long-Term Benefits of Chronic Azithromycin. Futhering Our Quest for Precision Medicine. Am J Resp Crit. Care Med. 2020, 15, 201 (4), 398-400.

19. Sala MA, Jain M .: Tezacaftor for treatment of cistic fibrosis. Expert Rev. Resp. Med. 2018, 12 (9), 725-732.

20. Souther KW, Barker PM: Azitromycin for cystic fibrosis. Eur. Resp. J. 2004, 24, 834835. 
21. Starner T., McCray P .: Pathogenesis of Early Lung Disease in Cystic Fibrosis. A Window of Oportunity to Eradicate Bacteria. Annals of Internal Medicine. ACP, 2005.

22. Szczeklik A .: Internal diseases. MP Krakow. 2010, 610-612.

23. Walkowiak J., Pogorzelski A., Sands D. et al .: Principles of diagnosis and treatment of cystic fibrosis. Poznań-Warsaw-Rzeszów. Medical Standards. 2009.

24. Zieliński J .: Genotype and phenotype in cystic fibrosis. Respiration. 2000, 67 (2), 117-133. 